\title{
Stable Multiple Myeloma or Plasma Cell Leukemia
}

National Cancer Institute

\section{Source}

National Cancer Institute. Stable Multiple Myeloma or Plasma Cell Leukemia. NCI

Thesaurus. Code C70642.

Stable multiple myeloma or plasma cell leukemia is characterized by not meeting the criteria for complete response, very good partial response, partial response or progression of disease. Stable disease requires two consecutive assessments made at any time before the institution of any new therapy, and no known evidence of progressive or new bone lesions if radiographic studies were performed; radiographic studies are not required to satisfy stable disease requirements. 